\title{
Bioconversion of Plant Raw Materials in Value-Added Products by Lentinus edodes (Berk.) Singer and Pleurotus spp.
}

\section{Giorgi Songulashvili, ${ }^{1,3}$ Vladimir Elisashvili, ${ }^{1}$ Michel Penninckx, ${ }^{2}$ Eka Metreveli, ${ }^{1}$ Yitzhak Hadar, ${ }^{4}$ Nana Aladashvili, ${ }^{1}{ }^{\circ}$ Mikheil Asatiani ${ }^{1}$}

${ }^{1}$ Durmishidze Institute of Biochemistry and Biotechnology, 0159 Tbilisi, Georgia; ${ }^{2}$ Universite Libre de Bruxelles, B-1180 Brussels, Belgium; ${ }^{3}$ Institute of Evolution, University of Haifa, Haifa 31905, Israel; ${ }^{4}$ Department of Plant Pathology and Microbiology, Faculty of Agricultural, Food and Environmental Quality Sciences, The Hebrew University of Jerusalem, Rehovot 76100, Israel

Lentinus edodes and Pleurotus spp. were compared for their ability to produce extracellular hydrolytic and oxidative enzymes in solid-state fermentation (SSF) and submerged fermentation (SF) of different lignocellulosic agroindustrial wastes. From received data the following conclusions were drawn.

First, fungi cultivation in identical culture conditions reveals wide differences among both species and strains of the same species. For example, in SSF of tree leaves by fungi of genus Pleurotus, endoglucanase activity varied from $0.5 \mathrm{U} \mathrm{mL}^{-1}(P$. ostreatus IBB 8) to $26.0 \mathrm{U} \mathrm{mL}^{-1}$ (P. ostreatus (Jacq.: Fr.)P. Kumm. IBB 2191), whereas xylanase activity varied from $3.1 \mathrm{U} \mathrm{mL}^{-1}$ (P. tuberregium (Rumph.: Fr.) Singer IBB 624) to $43.7 \mathrm{U} \mathrm{mL}^{-1}$ (P. ostreatus 2175).

Second, the nature and chemical composition of lignocellulosic material is one of the main factors determining the expression of fungi lignocellulolytic potential as well as the ratio of individual enzymes in lignocellulolytic system. For example, $P$. dryinus (Pers.:Fr.) P.Kumm. IBB 903 maximal laccase activity was equal to $119,195,794$, and $4103 \mathrm{U} / \mathrm{L}^{-1}$ in SF of milled tree leaves, banana, apple, and mandarin peels, respectively.

Third, the effect of lignocellulosic substrate concentration on enzyme synthesis varies in dependence on fungi peculiarities. Whereas P. dryinus IBB 903 hydrolytic and oxidative enzyme activity increased by 3-8 times with the elevation of mandarin peels or tree leaves concentration from 1 to $4-6 \%$, in the case of $P$. tuberregium IBB 624 the lowest substrate concentration was sufficient to ensure the maximal production of laccase, cellulase, and xylanase by this mushroom.

Fourth, the data proved that most plant raw materials tested contained sufficient nitrogen concentrations to ensure both mushroom growth and enzyme synthesis. However, depending on fungi and lignocellulosic substrate, supplementation of additional nitrogen sources in the medium effects the yield of lignocellulolytic enzymes. It is worth noting that in most cases only increased fungi growth and biomass in the presence of additional nitrogen accounts for the higher level of laccase activity.

Fifth, supplementing the culture medium by stimulators (xylidine, copper, or manganese) increased the yield of P. dryinus IBB 903 ligninolytic enzymes or caused their early and rapid accumulation in culture liquid.

The investigation of fruiting body yield and hydrolytic and oxidative enzyme accumulation during bioconversion of different lignocellulosic wastes by Pleurotus spp. and Lentinus edodes showed that bio- 
logical efficiency reached $86-118 \%$ and $81-116 \%$, depending on growth substrate and medium used to soak the substrate.

Cultivation of two strains of P. ostreatus on wheat straw and tree leaves as growth substrates clearly showed physiological and biochemical changes taking place during mushroom growth and development on plant raw materials. Mushroom cellulases and xylanase activity appeared to be very low during the vegetative phase of substrate colonization by mycelia. Their activity sharply rose with the appearance of primordia and reached their maximum at a stage of matured fruiting bodies. After fruit bodies were harvested, when the culture was in vegetative phase of development, the activity of these enzymes decreased again. In contrast, mushroom cultures were distinguished by high laccase and $\mathrm{MnP}$ activity during substrate colonization. The activity of both enzymes decreased at stages of primordia appearance and fruiting bodies maturation. However, when the culture enters the second vegetative phase (5 days after the fruit bodies were harvested), laccase and $\mathrm{MnP}$ activities increased again.

The study of lignocellulolytic enzyme activity of L. edodes in mushroom cultivation on wheat straw and tree leaves showed the same regularities that were revealed in cultivation of Pleurotus spp. with insignificant variations. The vegetative growth of these edible mushrooms is characterized by comparatively low activity of polysaccharases and high activity of ligninolytic enzymes. During the period of fruiting body formation, when the mushroom growth requires large quantities of plastic materials and energy, the secretion of cellulases and xylanase sharply increases to accelerate polysaccharide hydrolysis. In contrast, ligninolytic enzyme production appears, to some extent, is repressed to prevent the expenditure of constructive materials and energy.

Finally, the data on mushroom enzyme activity show that residual spent substrate may become a cheap source of lignocellulolytic enzymes for several biotechnological applications. 